$$
\text { تقيّم المعايير الزمنية لـهيدروكر اف الجريان الربيعي لنهر الزاب الاصغز }
$$

\title{
الخلاصة
}

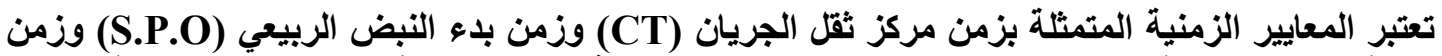

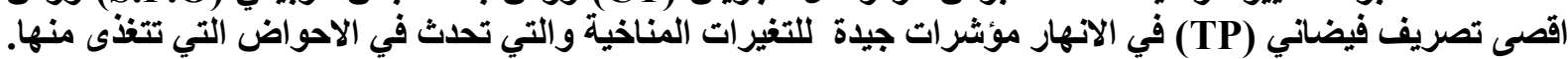

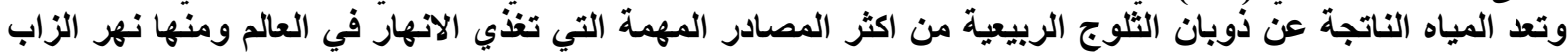

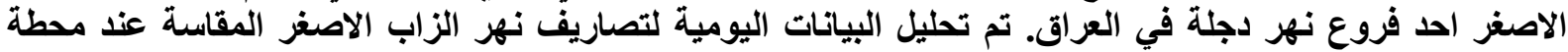

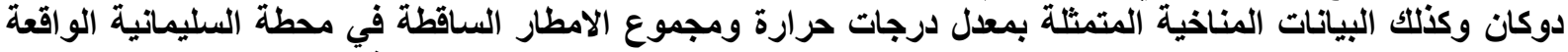

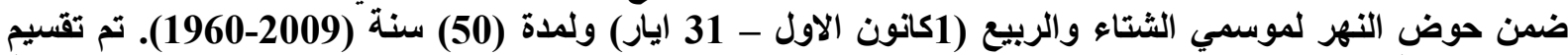

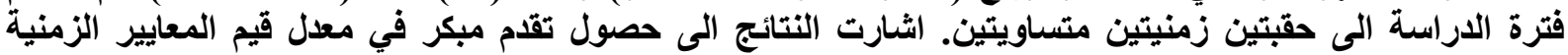

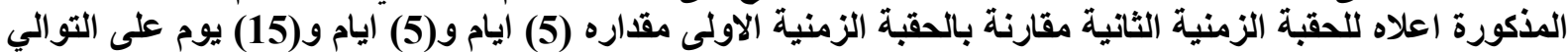

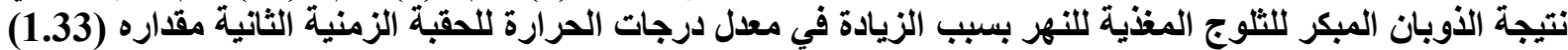

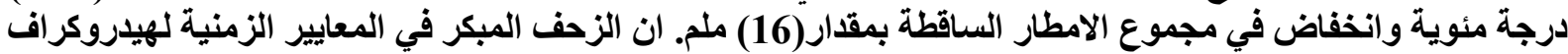

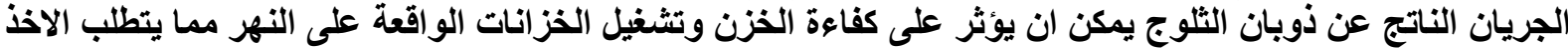
بنظر الاعتبار هذه التغيرات الهيدرولوجية في مشاريع الموارد التود المائية.

\section{Assessment Timing Criterions of Spring Streamflow Hydrograph for Lesser Zab River}

\author{
Mohanad T. AL-Sha`ar
}

\author{
Thair M. AL-Taiee
}

\begin{abstract}
The timing criterions represented by the time center of mass flow (CT), spring pulse onset (S.P.O) and time of flood peak discharge (TP) of rivers are considered good indicators for the climate change occurs on their watershed areas. Spring snowmelt is a main water resources for large number of rivers in the world such as lesser Zab river in Iraq. An analysis for the daily discharge of lesser Zab measured at Dokan station and for climatic data such as temperature and rainfall measured at Alsulaymania meteorological station is carried out for spring and winter seasons of the 50 water years (1960-2009) after dividing this study period into two equal consecutive eons. The results indicate an early advance for the average values of these above criterions in the second eon by 5, 5 and 15 days respectively in-comparison with the first eon as a result of early snowmelt feeding the river due to increasing temperature in the second eon $(1.33)^{\circ} \mathrm{c}$ and decreasing rainfall (16) $\mathrm{mm}$. The early advance in the timing criterions of the flow hydrograph occur from snowmelt may effect on the storage efficiency and operation of reservoirs located on the river and require taking these hydrological changes into consideration in the water resources projects.
\end{abstract}

Keywords: Climate Change, Spring Pulse, Lesser Zab, Streamflow, Early Advance 


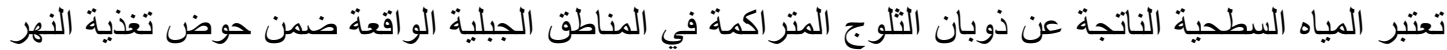

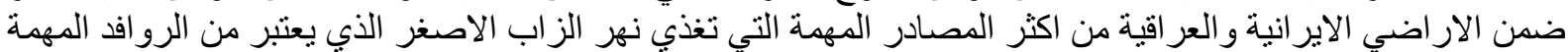

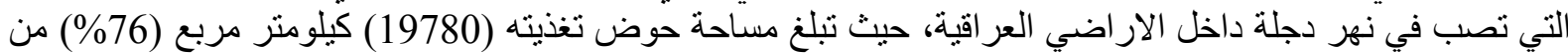

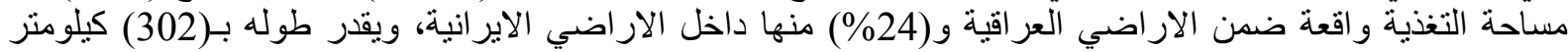

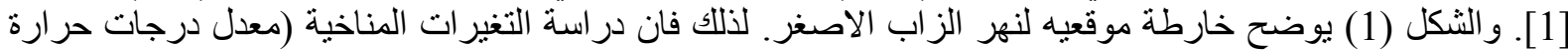

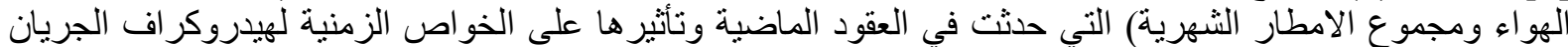

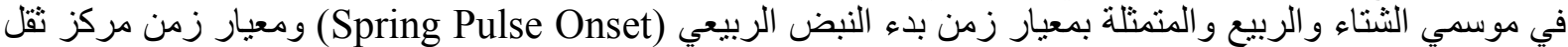

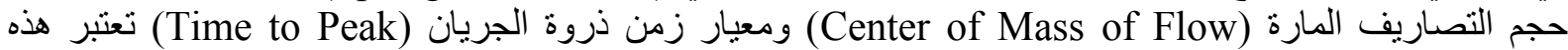

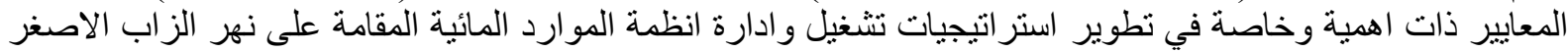

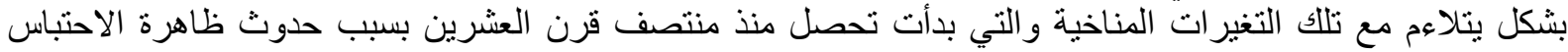

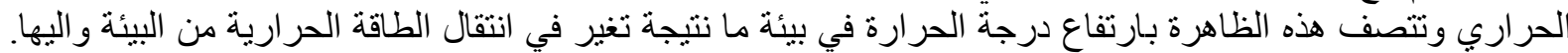

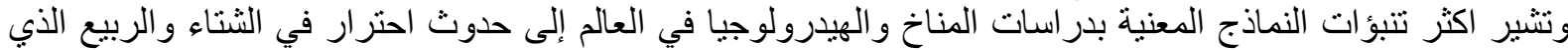

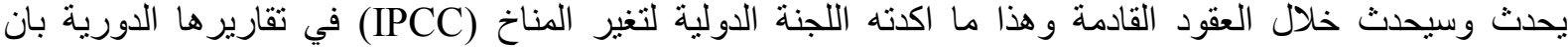

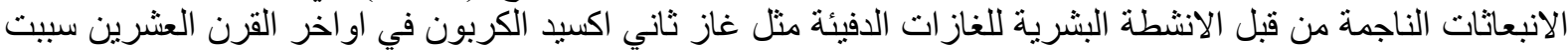

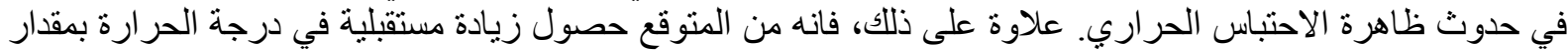

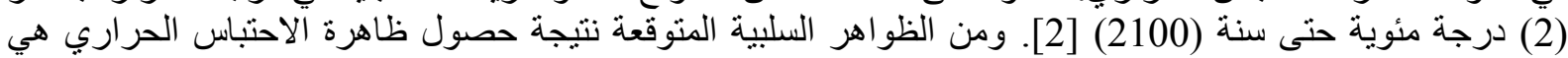

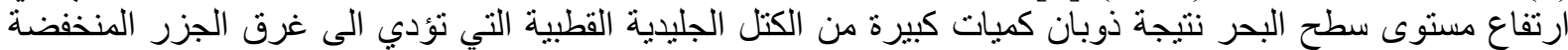

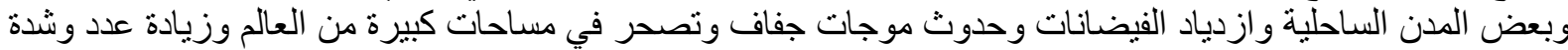

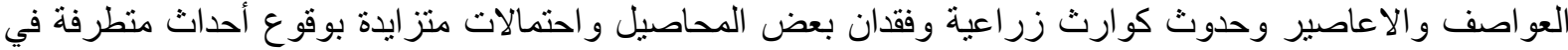
الطقس و انقر اض العديد من الكائنات الحية.

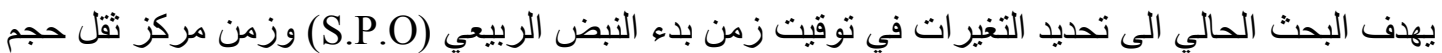

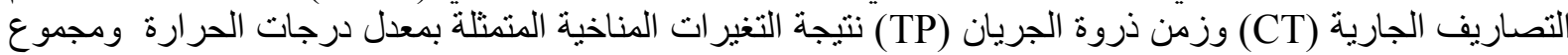
الامطار الثهرية.

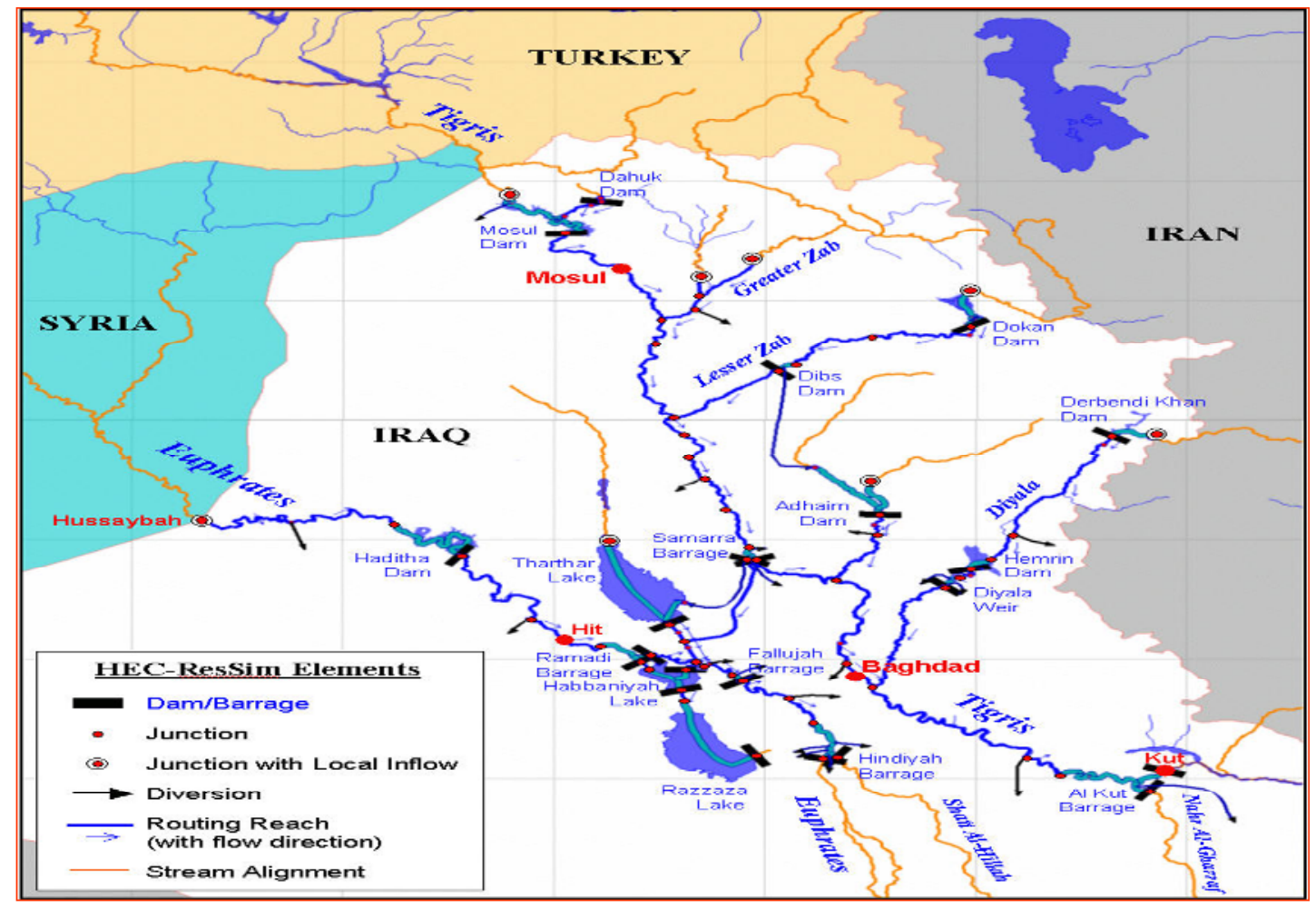

الثكل (1): خارطة موقعيه لنهر الزاب الاصغر ومساحته المغذية. 
تم الحصول على التصاريف اليومية الطبيعية لنهر الز اب الاصغر والمقاسة عند محطة دوكان الواقعة على نهر

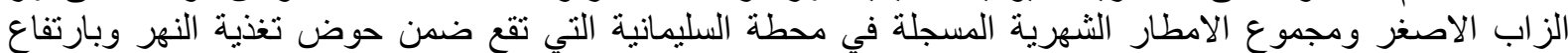

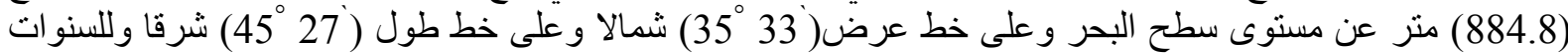
المائية (1960-2009) اما بالنسبة لمعدل درجات الحرارة الثهرية المسجلة في نفس المحطة فقد تم الحصول عليها اللسنو ات المائية (1972-2009).

\section{تحليل التصاريف اليومية:}

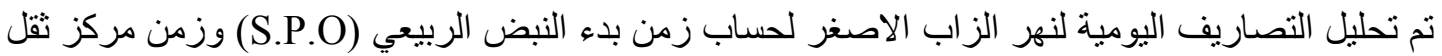

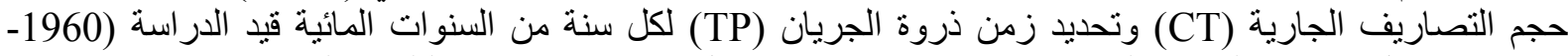

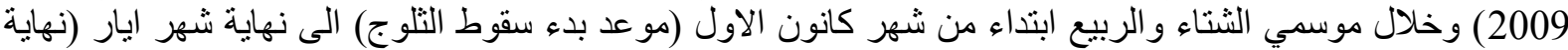

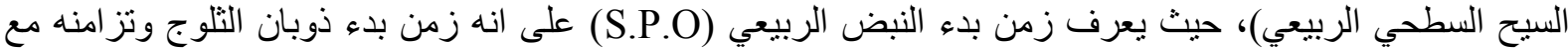

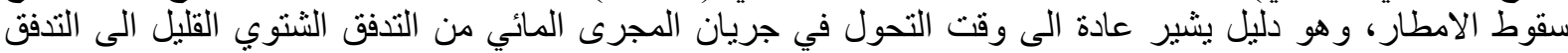

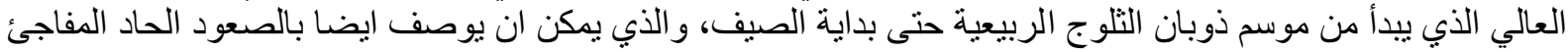

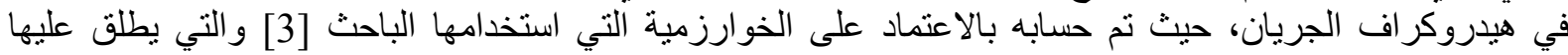

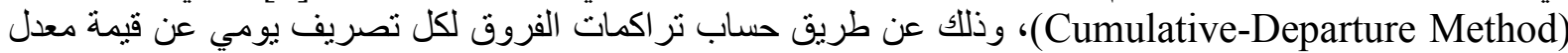

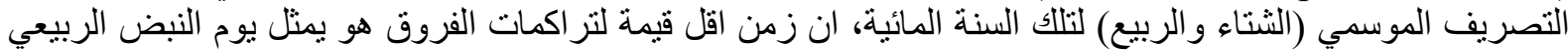

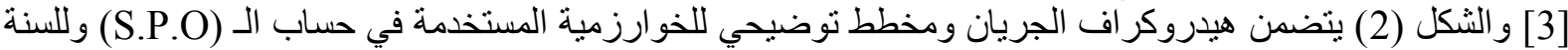

المائية (1971-1971).

يعرف زمن مركز ثقل حجم التصاريف اليومية (CT) بانه انعكاس للظروف المناخية التي تحكم زمن السيح

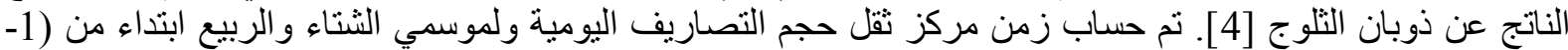

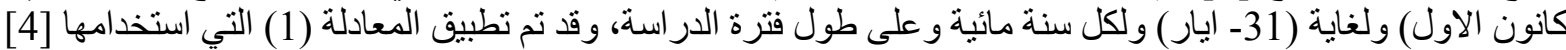
في حساب قيمة (CT) (CT)

$C T=\sum_{i=1}^{i=n}(t i \times q i) / \sum_{i=1}^{i=n} q i$

n n: عدد الايام ابتداء من (1- كانون الاول) ولغاية (30- ايار).

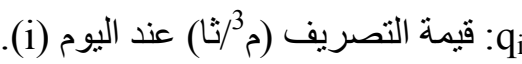

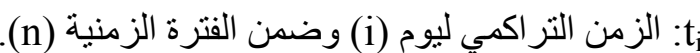

ان قيمة (n) تكون مساوية لـ (183) يوم اذ كانت السنة كبيسة وتساوي (182) يوم للسنة الغير كبيسة.

تم تحديد زمن ذروة التصريف الربيعية (TP) لكل السنوات المائية قيد الدراسة، وسميت بالذروة الربيعية لان النيان

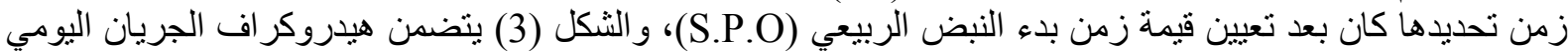

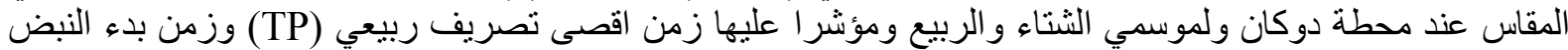

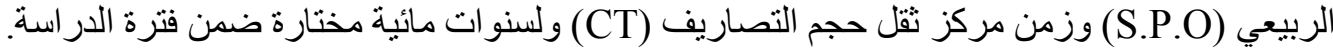



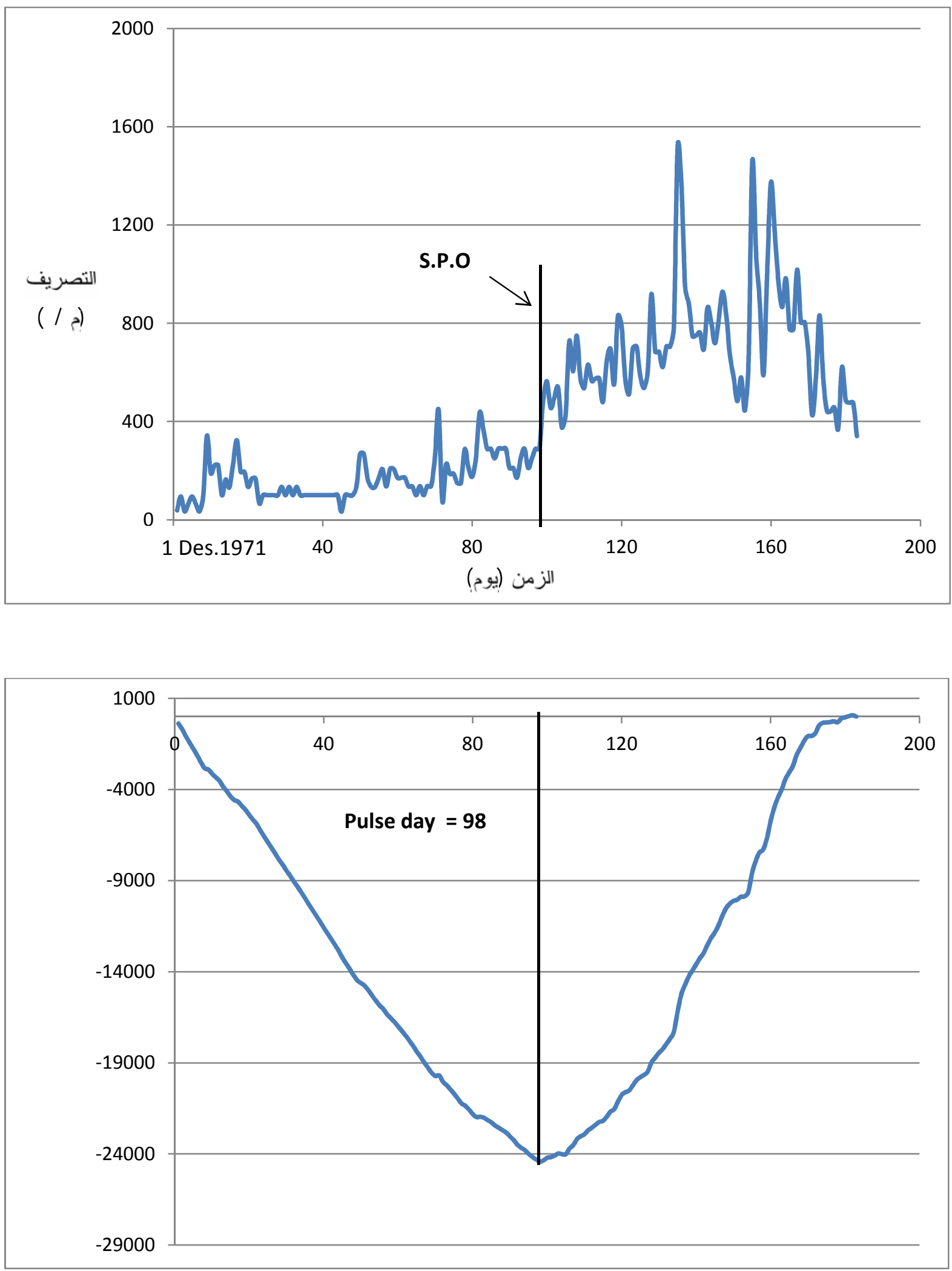

الثكل (2): هيدروكر اف الجريان الموسمي وخوارزمية تعيين يوم النبض الربيعي للسنة المائية (1971-1972). 
الطائي: تقييم المعايير الزمنية لهيروكراف الجريان الربيعي لنهز الزاب الاصفر

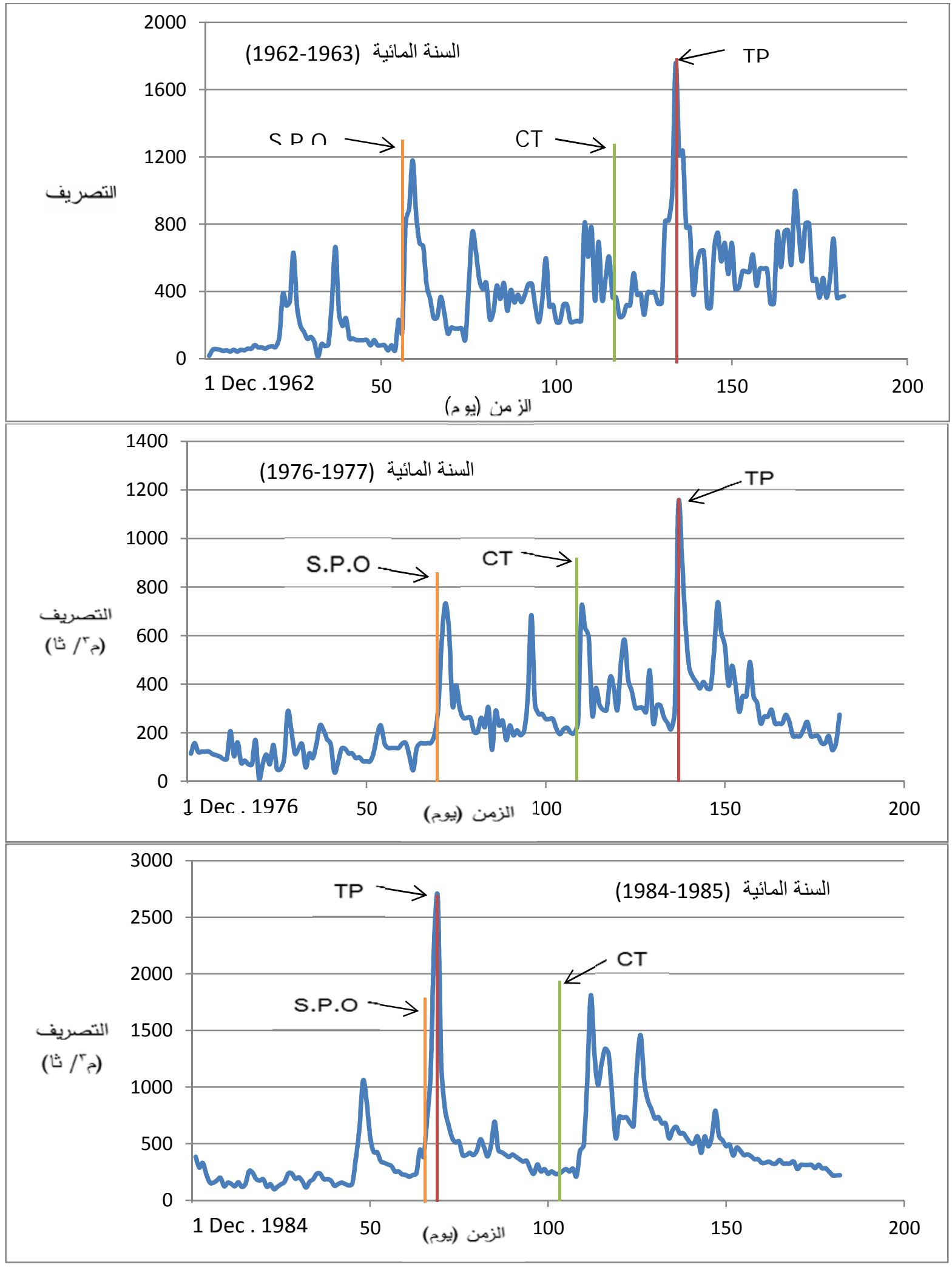

الثكل (3):هيدروكر اف الجريان اليومي لنهر الزاب الاصغر خلال موسمي الثتاء و الربيع ولسنوات مائية مختارة ضمن فترة الدر اسة 


\section{تحليل البيانات المناخية:}

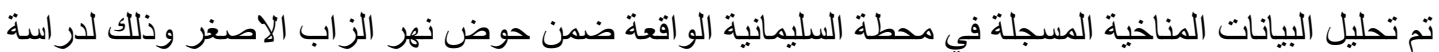

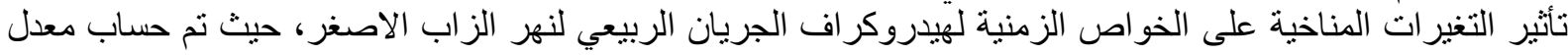

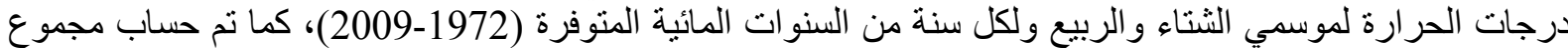
الامطار المسجلة في محطة السليمانية ولموسمي الثنتاء والربيع ايضا ولكل سنة من السنوات المينة المئية المنوفرة (1960-

بعد تقسيم فترة الدراسة و التي تضمنت تحليل التصاريف اليومية الداخلة الى محطة دوكان و الو اقعة على نهر

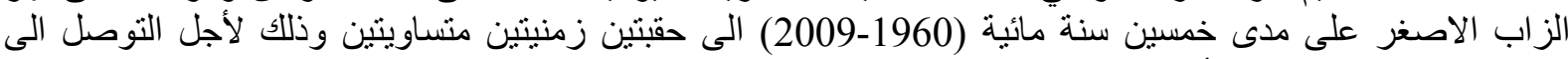

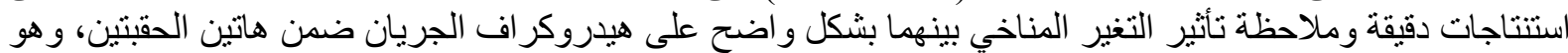

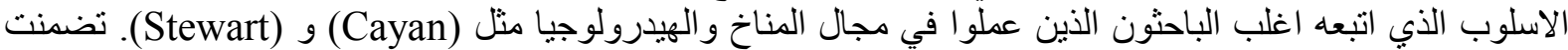

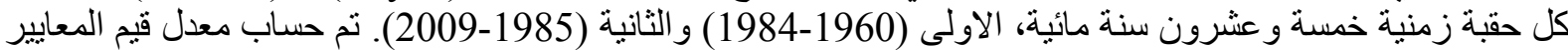
الزمنية (S.P.O , CT , TP) لكل حقبة زمنية وبعد مقارنة معدل قيم هذه المعايير للحقبتين الزمنيتين اظهرت التهية النتائج

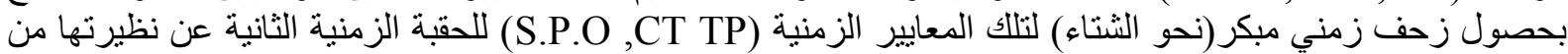

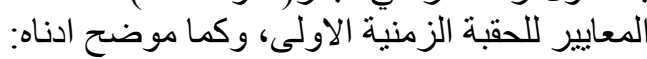

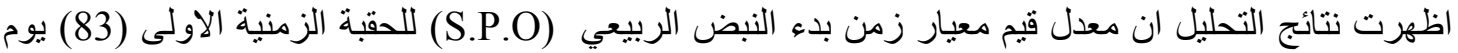

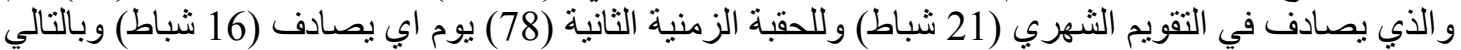

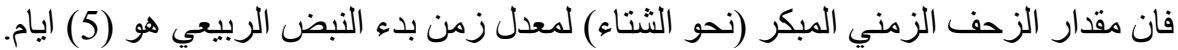

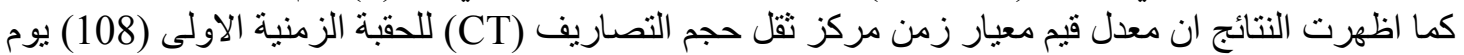

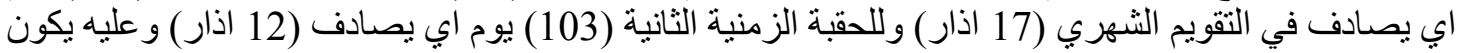

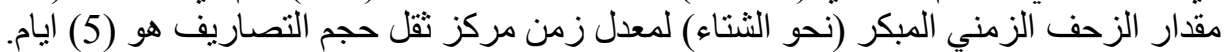

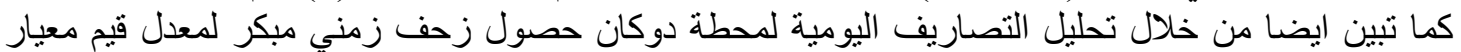

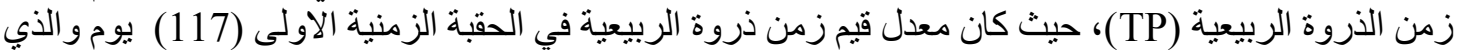

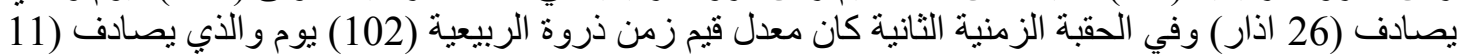

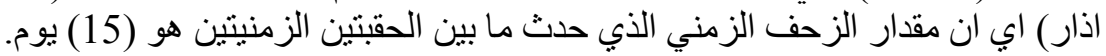

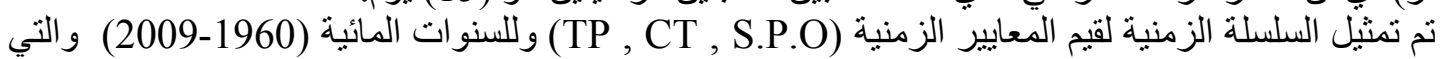

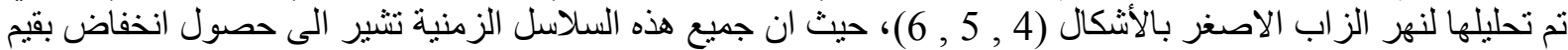
المعايير الزمنية (TP , CT , S.P.O) و على طول السنوات الات المائية قيد الدراسة.

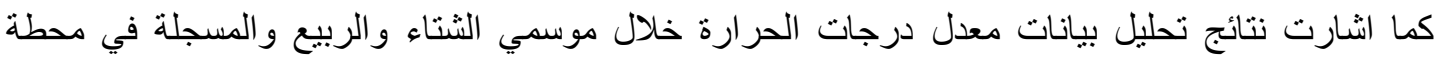

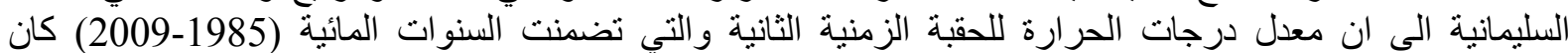

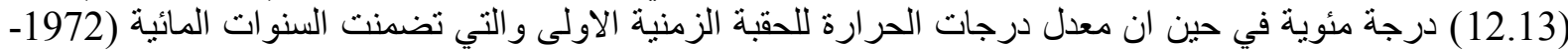

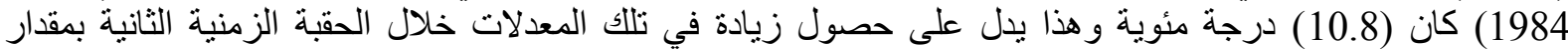

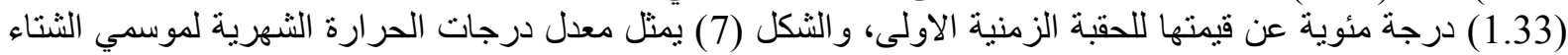

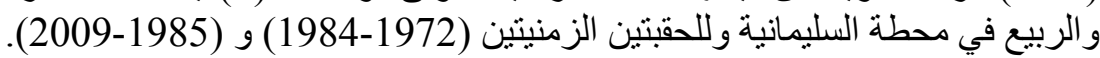

تم تحليل مجموع الامطار الساقطة خلال موسمي الثتاء و الربيع و المقاسة في محطة السليمانية وللسنوات المائية المئية (2009-1960) حيث قسمت فترة التحليل الى حقبتين زمنيتين، الحقبة الزمنية الاولى (1960 (1964-1984) و الحقبة الزمنية فئية

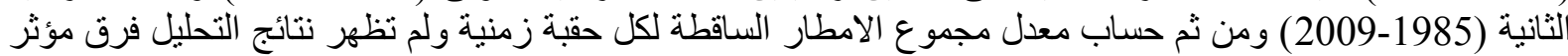

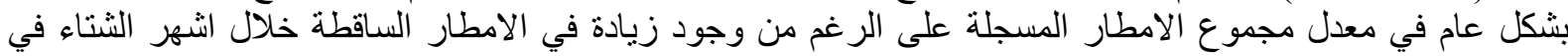

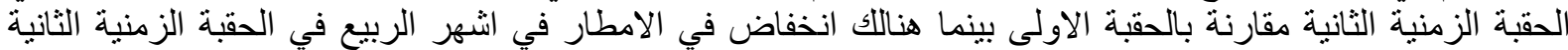

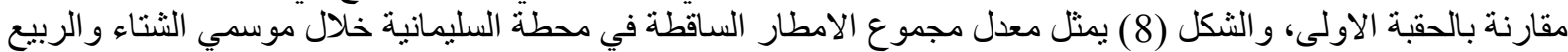
وللحقبتين الزمنيتين (1960-1984) و (1985-2009). 
الطائي: تقييم المعايير الزمنية لهياروكراف الجريان الربيعي لنهز الزاب الاصغر

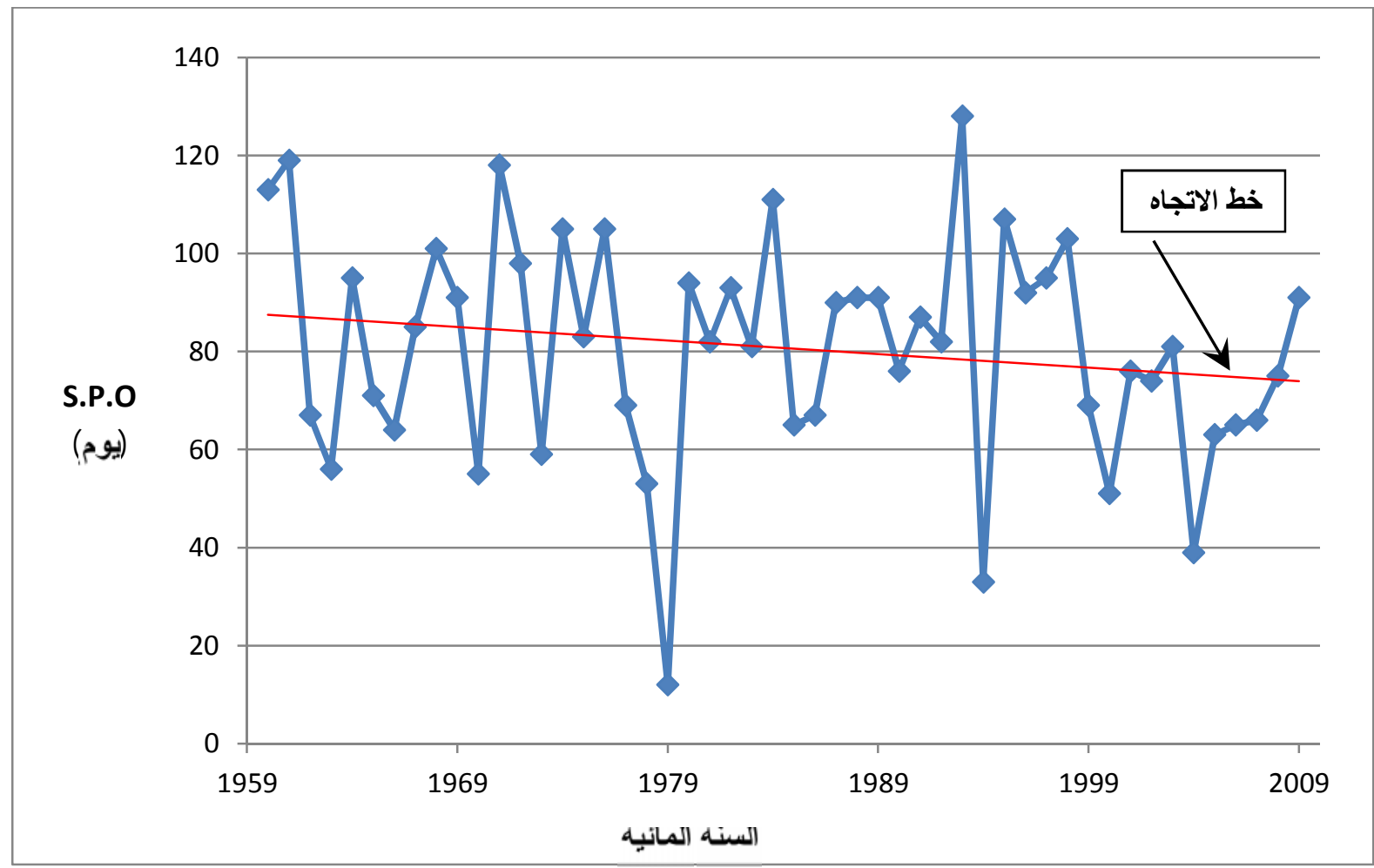

الثكل (4): سلسلة زمنية لقيم S.P.O لنهر الزاب الاصغر وللسنوات المائية (1960-2009).

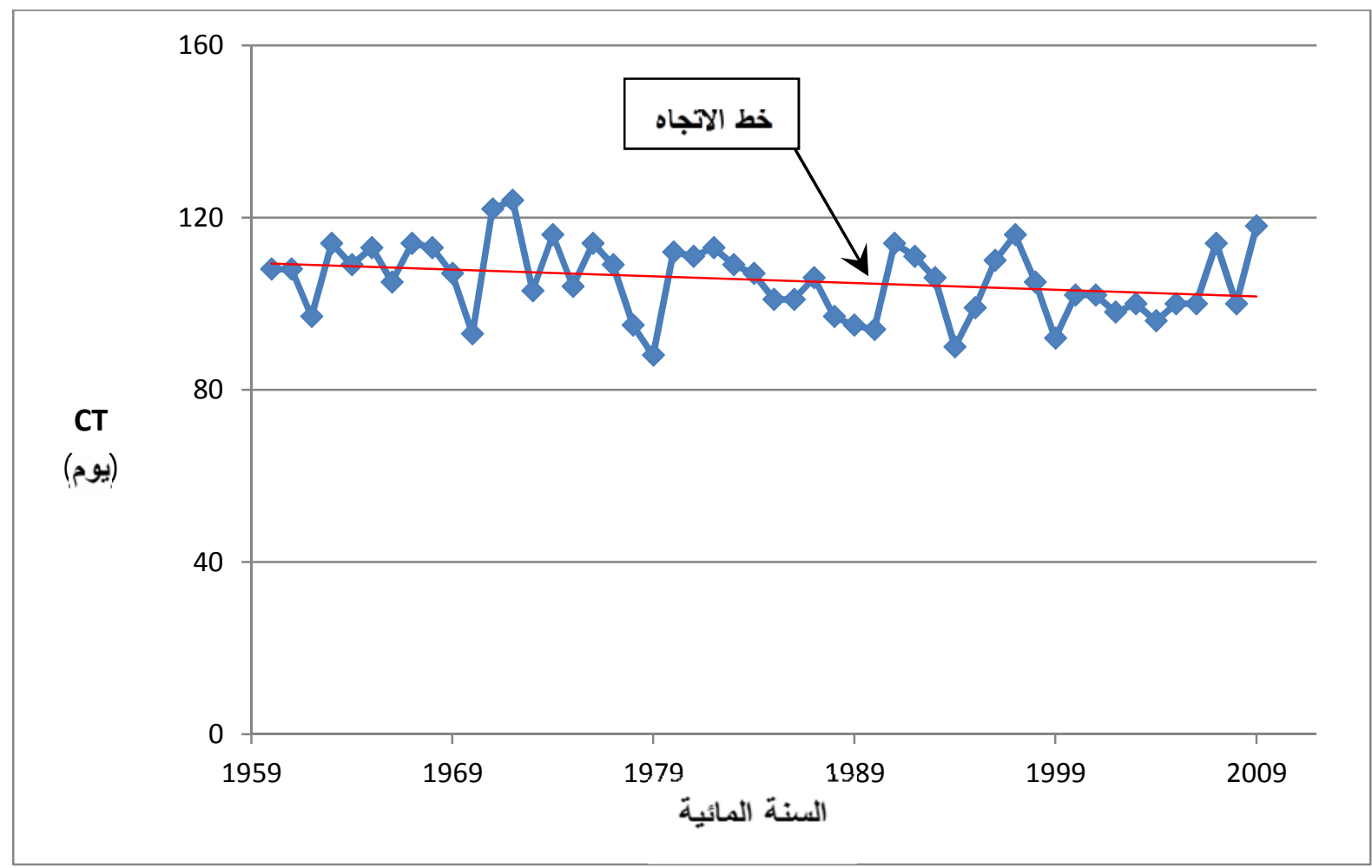

الثكل (5): سلسلة زمنية لقيم CT لنهر الزاب الاصغر وللسنوات المائية (1960-2009). 


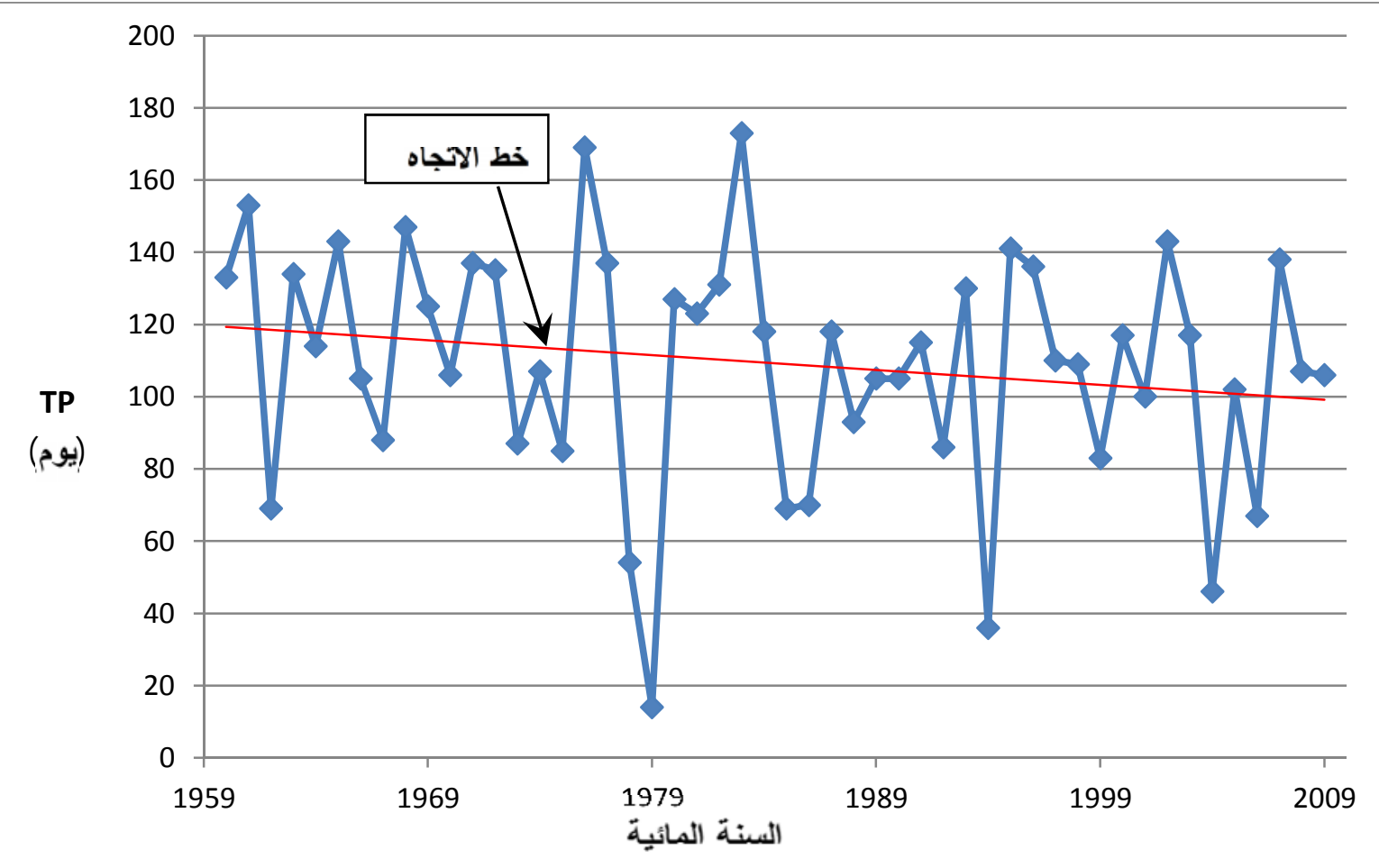

الثكل (6):سلسلة زمنية لقيم TP لنهر الزاب الاصغر وللسنوات المائية(1960-2009).

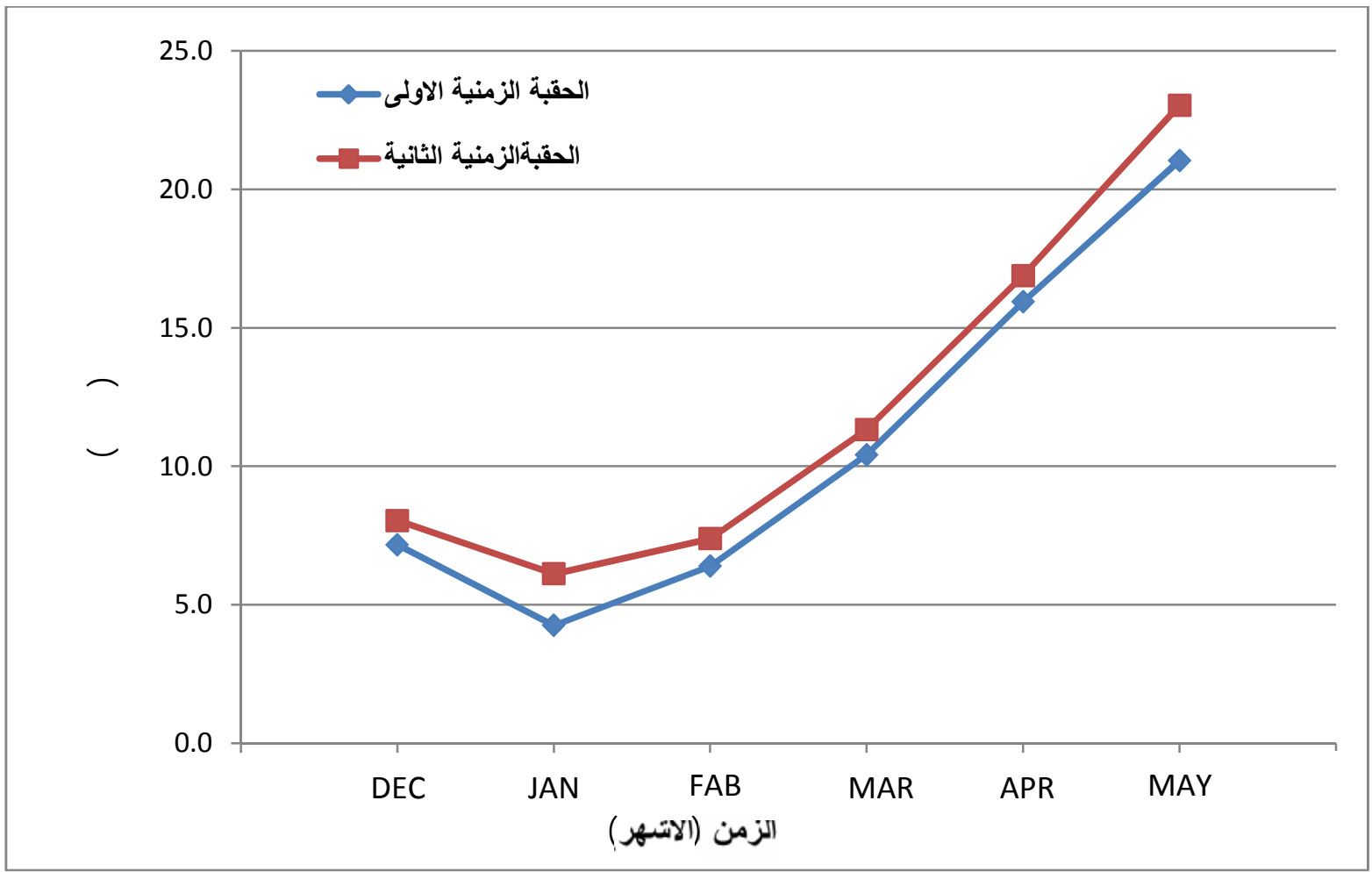

الثكل (7): معدل درجات الحرارة لموسمي الثتاء و الربيع المسجلة في محطة السليمانية خلال حقبتي الدر اسة (1984-1972) و (2009-1985) 
الطائي: تقييم المعايير الزمنية لهيروكراف الجريان الربيعي لنهر الزاب الاصغر

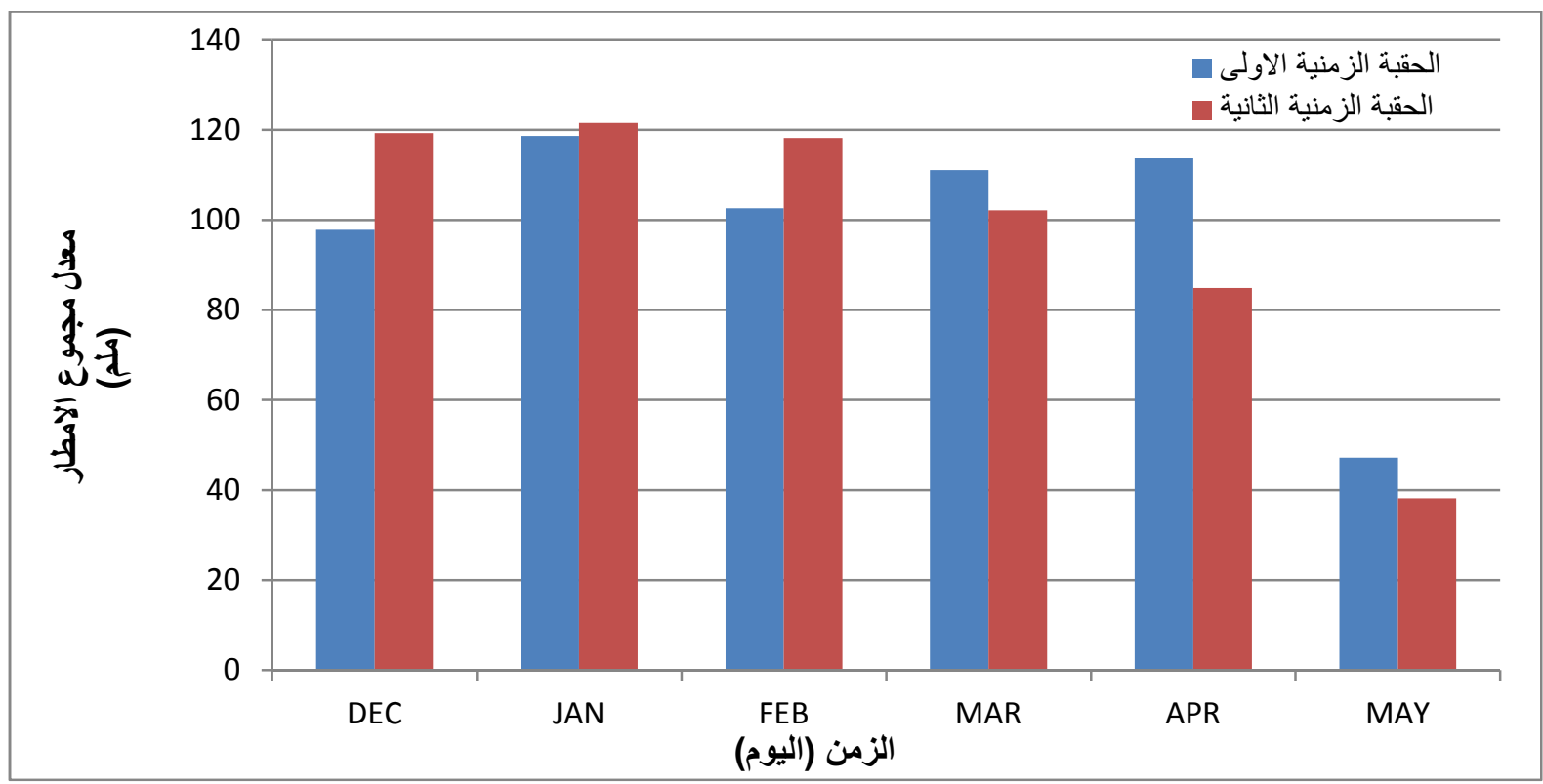

الثكل (8): معدل مجموع الامطار الساقطة في محطة السليمانية خلال موسمي الثتاء و الربيع وللحقبتين الزمنيتين (1984-1960) و (1985)

الاستتناجات والمقترحات:

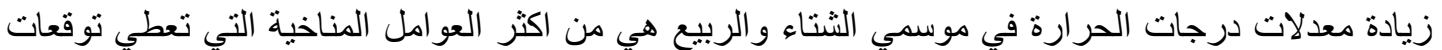

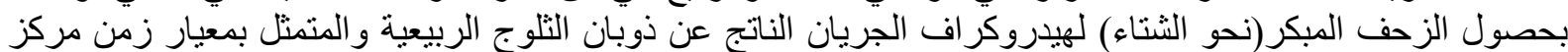

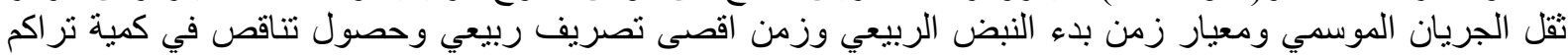

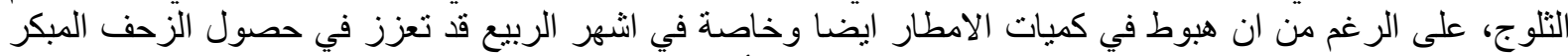

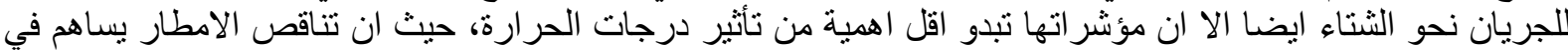

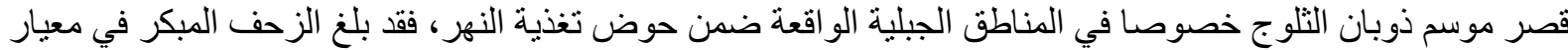

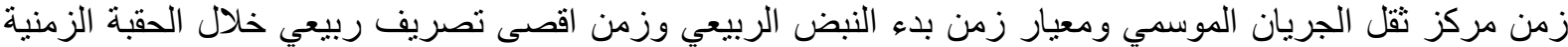

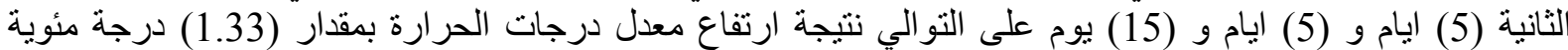

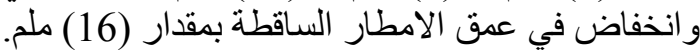

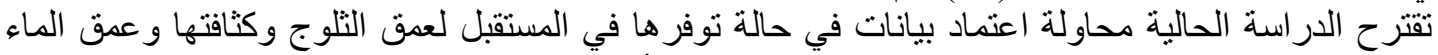

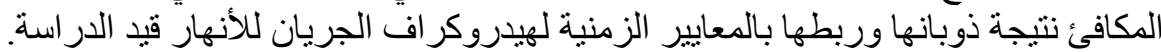

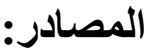

1- ESCWA, (2012)."Inventory of Shared Water Resources in Western Asia".

2- Kenneth Frederick ,(1997)." Water Resources and Climate Change", Climate Issues Brief No. 3 .

3- Cayan, D. R., Kammerdiener, S. A., Dettinger, M. D., Caprio, J. M., and Peterson, D. H.,(2001)."Changes in the Onset of Spring in the Western United States", Bull. Amer. Meteorological.Soc. 82, 399-415.

4- Stewart, I.T., Cayan, D.R., and Dettinger, M.D.,(2004). "Changes in Snowmelt Runoff Timing in Western North America Under 'Business as Usual' Climate Change Scenario", Climate Change, 62: 217-232.

تم اجراء البحث في كلية ألهندسة = جامعة ألموصل 